\title{
Evaluación tribológica de recubrimientos de nbc y VC sobre acero AISI D2 producidos por la técnica deposición difusión termo-reactiva
}

\author{
Tribological Evaluation of Nbc and Vc Coatings on Steel AISI D2 Produced \\ by the Thermo-Reactive Deposition Difusion Technique
}

\author{
Ramírez-Ramos Marco Antonio \\ Departamento de Ingeniería Mecánica y Mecatrónica \\ Posgrado de Materiales y Procesos \\ Universidad Nacional de Colombia, Bogotá \\ Correo:maaramirezra@unal.edu.co \\ Olaya-Flores John Jairo \\ Departamento de Ingeniería Mecánica y Mecatrónica \\ Posgrado de Materiales y Procesos \\ Universidad Nacional de Colombia, Bogotá \\ Correo:jjolayaf@unal.edu.co
}

\author{
Trava-Airoldi Vladimir Jesús \\ Laboratorio Asociado de Sensores y Materiales LAS \\ Instituto Nacional de Investigaciones Espaciales de Brasil INPE \\ Correo:vladimir@las.inpe.br
}

Información del artículo: recibido: febrero de 2014, reevaluado: marzo de 2014, aceptado: mayo de 2014

\section{Resumen}

En este trabajo se obtuvieron recubrimientos de carburos de vanadio (VC) y carburos de niobio $(\mathrm{NbC})$ sobre aceros para herramientas de la norma AISI D2 usando el sistema de deposición difusión termo reactiva (DTR). Los experimentos se realizaron en baño de sal (bórax $\mathrm{Na}_{2} \mathrm{~B}_{4} \mathrm{O}_{7}-10 \mathrm{H}_{2} \mathrm{O}$ ) con disolución de ferro-niobio, ferro-vanadio y aluminio a una temperatura de tratamiento de $1000^{\circ} \mathrm{C}$ durante 3 horas. Los recubrimientos obtenidos se caracterizaron mediante microscopía electrónica de barrido (MEB) y difracción de rayos $\mathrm{X}(\mathrm{DRX})$. El comportamiento tribológico se estudió por la técnica de esfera sobre disco para determinar el coeficiente de fricción (COF) y la técnica de rayado para determinar el nivel adherencia. Los resultados se compararon con los substratos sin recubrimiento, el recubrimiento logró disminuir el coeficiente de fricción y aumento en la dureza del sistema debido a su naturaleza cerámica.

\section{Descriptores:}

- carburo de niobio

- carburo de vanadio

- desgastecoeficiente de fricción deposición difusión termo-reactiva

- recubrimientos 


\begin{abstract}
In this work vanadium carbides $(\mathrm{VC})$ and niobium carbide $(\mathrm{NbC})$ coatings were produced on AISI D2 tool steels system using the thermo reactive deposition diffusion technique (TRD). The experiments were performed in a salt bath (borax) with dissolution of ferro-niobium, ferro-vanadium and aluminium at temperature treatment of $1000^{\circ} \mathrm{C}$ during 3 hours. The coatings obtained were characterized by scanning electron microscopy and X-ray diffraction. The tribological behavior was studied by the technique of pin on disc to define the coefficient of friction and scratch technique to determine the adhesion level. Results were compared with uncoated substrates, a decrease of the friction coefficient and an increase in the hardness was observed.
\end{abstract}

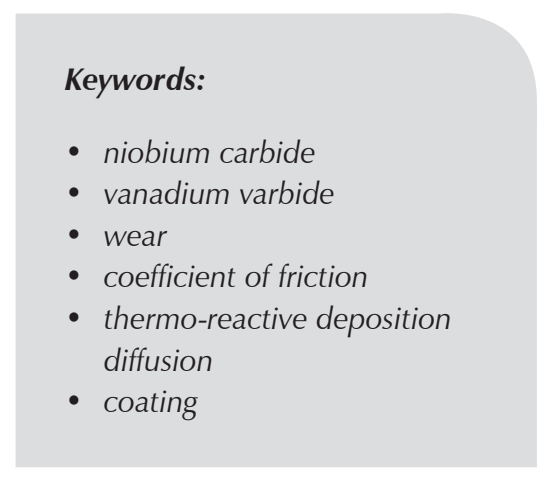

\section{Introducción}

El estudio de las propiedades mecánicas y tribológicas de los recubrimientos duros se hace indispensable para definir las aplicaciones en un determinado proceso tecnológico por lo que es necesario explorar sus diferentes posibilidades de acuerdo con su dureza, resistencia al desgaste y alta adherencia, entre otros. Capas duras y resistentes al desgaste se produjeron sobre aceros de tipo AISI D2 con concentración mayor a $0.3 \%$ de C, se obtuvieron por la técnica deposición difusión termoreactiva (DTR) (Arai 1991 y 1979). Estas capas presentan interfaces planas y definidas, alta adherencia con el sustrato y elevada dureza, además de un comportamiento óptimo al desgaste y bajos coeficientes de fricción. El tratamiento utilizado para este proceso fue un baño de sal (bórax) que contiene en disolución los elementos formadores de carburos (EFC's), ferro-niobio, ferro-vanadio y aluminio como agente reductor.

Los recubrimientos se forman por una combinación química de los átomos de $C$ presentes en la matriz del sustrato con los EFC's disueltos en el baño de bórax, debido a una baja energía libre de formación del carburo y una energía de formación del óxido superior presente en la reacción (De Paula y Peter, 2012). Estos elementos son óptimos para ese proceso y se usan con frecuencia para formar estos recubrimientos (Arai, 1979; Oliveira et al., 2006a).

En la investigación realizada, el resultado de estos recubrimientos estuvo marcado por la presencia de $\mathrm{Nb}$ y $\mathrm{V}$ en las capas formadas sobre los aceros. En el presente trabajo el acero D2 se utilizó para producir capas duras además de estudiar el comportamiento tribológico por medio de los diferentes mecanismos.

Los trabajos reportados de carburos metálicos crecidos mediante el proceso DTR, se han caracterizado por obtener resultados de resistencia al desgaste, de adherencia, dureza elevada y buen comportamiento mecánico. Existen varios trabajos enfocados a la producción de recubrimientos de $\mathrm{VC}$ y $\mathrm{NbC}$ sobre sustratos de aceros del tipo AISI H13, D2 y M2, reportando durezas de 2500 HV (De Paula y Peter, 2012; Arai, 1979; Oliveira et al., 2006a). Otras investigaciones han determinado la cinética de crecimiento de carburos de niobio sobre el acero AISI 1040 (Oliveira et al., 2006b), de boruros de hierro sobre acero AISI 4140 (Saduman, 2005) y de carburos de cromo sobre acero AISI D2 (Holmberg et al., 1998). Estos trabajos reportan comportamiento tribológico y cinéticas de crecimiento en función del tiempo y la temperatura del tratamiento.

El objeto de esta investigación fue realizar un estudio sobre la resistencia al desgaste, dureza y adherencia del recubrimiento de carburo de niobio y carburo de vanadio sobre aceros para herramientas de tipo AISI D2 crecidos mediante la técnica de DTR. La combinación de estos resultados permite recomendar la producción de recubrimiento carburos de $\mathrm{Nb}$ y de $\mathrm{V}$ con el sistema de DTR para mejorar la durabilidad del acero AISI D2.

\section{Parte experimental}

\section{Materiales}

Carburos a base de niobio y vanadio se depositaron sobre un acero para herramientas AISI D2, la composición química del acero se muestra en la tabla 1. Antes del tratamiento, se cortaron muestras en forma de disco de acero de $16 \mathrm{~mm}$ de diámetro y $5 \mathrm{~mm}$ de espesor. La preparación de la muestra se hizo mediante pulimento metalográfico con papel lija núm. 260, 320, 600, 1000, 1200, pasta de diamante de $0.125 \mu \mathrm{m}$, y limpieza por medio de acetona en ultrasonido. 
Tabla 1. Composición química del sustrato AISI D2

\begin{tabular}{cccccccccc}
\hline Composición & $\mathrm{C}$ & $\mathrm{Si}$ & $\mathrm{Mn}$ & $\mathrm{P}$ & $\mathrm{S}$ & $\mathrm{Cr}$ & $\mathrm{Ni}$ & $\mathrm{Mo}$ & $\mathrm{Fe}$ \\
\hline $\mathrm{Wt} \%$ & 1.48 & 0.102 & 0.414 & 0.025 & 0,032 & 11.91 & 0.027 & 0.98 & Balance \\
\hline
\end{tabular}

Las muestras se perforaron cerca del borde para colgarlas e introducirlas en el baño de bórax, se utiliza especialmente esta sal por su capacidad para disolver óxidos y su gran estabilidad térmica, bajo nivel evaporación, emisión de gases contaminantes y su bajo costo (Sen, 2004). En la tabla 2 se presenta la composición de los baños de sal utilizados durante el experimento. Se ha encontrado que a $1000^{\circ} \mathrm{C}$ el elemento formador de carburo se oxida con gran rapidez y forma oxido del metal agregado en la ferroaleación (vanadio o niobio), por lo tanto, no es posible obtener una capa de carburo; por esta razón se hace necesaria la utilización de un elemento reductor adicional que se encarga de reducir el bórax, evitar que se oxide el metal de transición aportado para favorecer la formación de los carburos deseados. El aluminio puro entre 1 y $10 \%$ (Patiphan y Prasonk) que se agrega en el baño de sales cumple el papel de agente reductor, es decir, que actúa como reductor del óxido de boro y se oxida para evitar que el metal agregado se oxide. De esta manera estos metales quedan libres para combinarse con el carbono del acero y formar carburos.

Tabla 2. Composición de los baños

\begin{tabular}{lcccc}
\hline Muestra & $\mathrm{Na} 2 \mathrm{~B} 4 \mathrm{O} 7$ & $\mathrm{Fe}-\mathrm{Nb}$ & $\mathrm{Fe}-\mathrm{V}$ & $\mathrm{Al}$ \\
\hline $\mathrm{D} 2-\mathrm{NbC}$ & $84 \%$ & $13 \%$ & 0 & $3 \%$ \\
$\mathrm{D} 2-\mathrm{VC}$ & $84 \%$ & 0 & $13 \%$ & $3 \%$ \\
\hline
\end{tabular}

El tratamiento se realizó a una temperatura de $1000^{\circ} \mathrm{C}$ por un tiempo de permanencia de $3 \mathrm{~h}$, con un precalentamiento de las muestras a una temperatura de $650^{\circ} \mathrm{C}$ por 0.5 h (Arai, 1998). La figura 1 muestra el ciclo térmico que se utilizó durante el crecimiento de los recubrimientos. Antes de procesar las piezas, es importante hacer un precalentamiento con el fin de evitar distorsiones y reducir el tiempo del proceso. Se recomienda usar la temperatura de austenización del acero que se esté tratando, para poder realizar el proceso de temple en aire, sal o aceite inmediatamente después del tratamiento DTR y por último, un tratamiento térmico de revenido para ajustar la dureza del sustrato.

Las fases presentes y las orientaciones se determinaron utilizando difracción de rayos $X$, en un equipo $X$ Pert Pro Panalytical operando a $45 \mathrm{kV}$ y $40 \mathrm{~mA}$, en un intervalo de 20 a $90^{\circ}$ y tamaño de paso de $0.02^{\circ}$, con una radiación monocromática CuK $\alpha$ de $1.594 \AA$ de longitud de onda. El espesor de los recubrimientos obtenidos se midió utilizando un perfilómetro óptico WYKO NT1100. Los recubrimientos se analizaron mediante microscopía electrónica de barrido, marca JEOL con capacidad de análisis por energía dispersiva por rayos $\mathrm{X}$.

Ensayos de esfera sobre disco

Para la prueba de esfera sobre disco se utilizó un tribómetro CETR-UM2 con contra cuerpo esférico compuesto por $95 \%$ de $\mathrm{Al}_{2} \mathrm{O}_{3}$, diámetro de $4 \mathrm{~mm}$ y dureza de $1610 \pm 101$ (HV 0.2). Los parámetros usados en la prueba de desgaste se muestran en la tabla 3.

Tabla 3. Parámetros utilizados para prueba de esfera sobre disco

\begin{tabular}{lc}
\hline \multicolumn{1}{c}{ Parámetros } & Valores \\
\hline Carga Aplicada $(\mathrm{N})$ & 4 \\
Velocidad $(\mathrm{m} / \mathrm{s})$ & 0.125 \\
Temperatura $\left({ }^{\circ} \mathrm{C}\right)$ & 19 \\
Humedad $(\%)$ & 83 \\
Tiempo $(\mathrm{s})$ & 480 \\
\hline
\end{tabular}

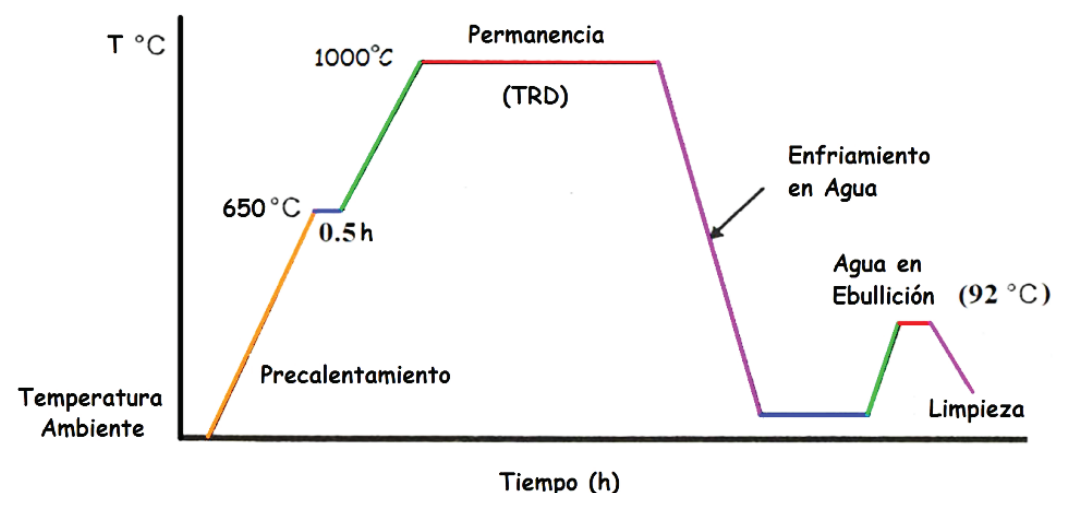

Figura 1. Ciclo térmico utilizado en el proceso DTR 
Ensayos de adherencia

Para esta prueba de adherencia se utilizó un tribómetro CETR-UM2 con contra cuerpo cónico en punta de diamante indentador Rockwell C de $200 \mu \mathrm{m}$ de radio, velocidad de desplazamiento de $60 \mathrm{~mm} / \mathrm{min}$. Los parámetros usados en la prueba de rayado están determinados por la carga aplicada variando de 0 hasta $80 \mathrm{~N}$ e incrementando proporcionalmente esta carga a lo largo del desplazamiento, el tiempo fue de $50 \mathrm{~s}$ para todas las pruebas realizadas.

\section{Ensayos de dureza}

La dureza de los recubrimientos se determinó a partir de mediciones de nanoindentación utilizando un equipo CETR UMT-1. La tabla 4, muestra los parámetros utilizados.

Tabla 4. Parámetros utilizados para medidas de nanodureza

\begin{tabular}{lc}
\hline \multicolumn{1}{c}{ Parámetros } & Valores \\
\hline Velocidad de aproximación $(\mathrm{nm} / \mathrm{min})$ & 2000 \\
Tipo de carga & Lineal \\
Incremento de carga $(\mathrm{nm} / \mathrm{min})$ & 60 \\
Carga máxima $(\mathrm{nm})$ & 30 \\
\hline
\end{tabular}

\section{Resultados y discusión}

La figura 2 presenta el difractograma obtenido de los recubrimientos de $\mathrm{NbC}, \mathrm{VC}$, producidos sobre acero AISI D2. Los recubrimientos presentaron una estructura FCC con una orientación mixta en los planos (111),
(200) y (222). También se aprecia la señal de Fe que corresponde a la fase cúbica del acero del sustrato (Oliveira et al., 2005). Se obtuvieron valores de dureza por medio de pruebas de nanoindentación para recubrimientos de $\mathrm{NbC}$ en un valor de $23.7 \mathrm{GPa} \pm 1.02$ con un módulo de elasticidad de $412 \mathrm{GPa}$, para recubrimientos de $\mathrm{VC}$ en un intervalo de $25.2 \mathrm{GPa} \pm 2.6$ y un módulo de elasticidad de $332 \mathrm{GPa} \pm 10.7$, de 2 a 4 veces la dureza de los sustratos debido al aumento de las microtensiones internas del recubrimiento.

Los recubrimientos obtenidos presentan una interfase plana y definida con el sustrato. La micrografía de sección transversal del acero AISI D2 para cada recubrimiento se muestra en la figura 3. El valor del espesor para el recubrimiento de $\mathrm{NbC}$ fue de $16.3 \mu \mathrm{m} \pm 1$. 6 y 15.8 $\mu \mathrm{m} \pm 0.9$ para el recubrimiento de $\mathrm{VC}$, respetivamente. No se presentaron variaciones significativas en el espesor, ya que el crecimiento dependió de la cantidad de carbono en la matriz y para este experimento el acero es homogéneo.

La figura 4 muestra el coeficiente de fricción (COF) de cada recubrimiento producidos en el ensayo de desgaste de esfera sobre disco. Se observa que el comportamiento tribológico cambia en el recubrimiento de $\mathrm{NbC}$ y VC sobre el acero D2 con relación al acero sin recubrir, con variaciones del COF desde 0.02 hasta 0.1 y con un comportamiento estable a lo largo del tratamiento. Para el acero D2 sin recubrir el valor obtenido del coeficiente de fricción es 0.35 , además la variación fue muy grande en la fricción durante la prueba y puede atribuirse a diferencias en la estructura de la película o irregularidades en la superficie debido a la rugosidad (Arai, 1991; Sen, 2005).
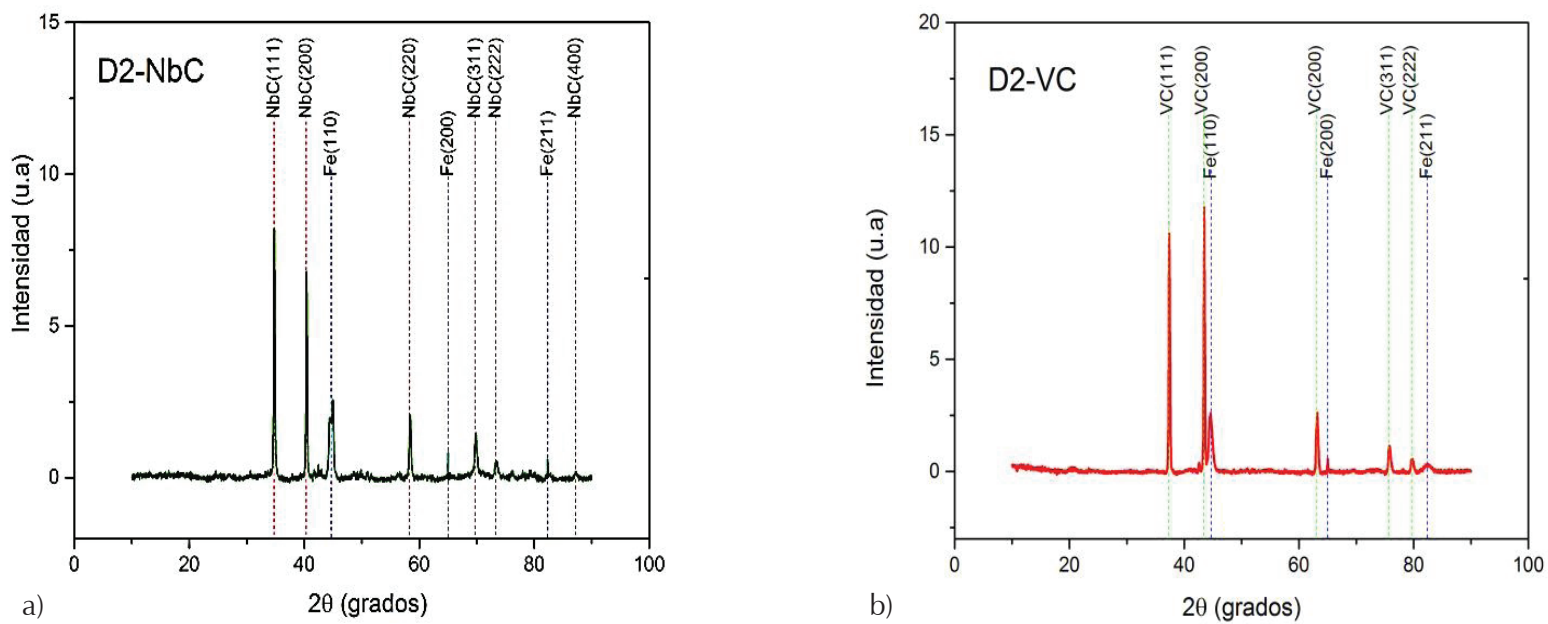

Figura 2. a) patrones de difracción rx de recubrimientos de carburos de niobio y b) vanadio, producidos sobre acero AISI D2 

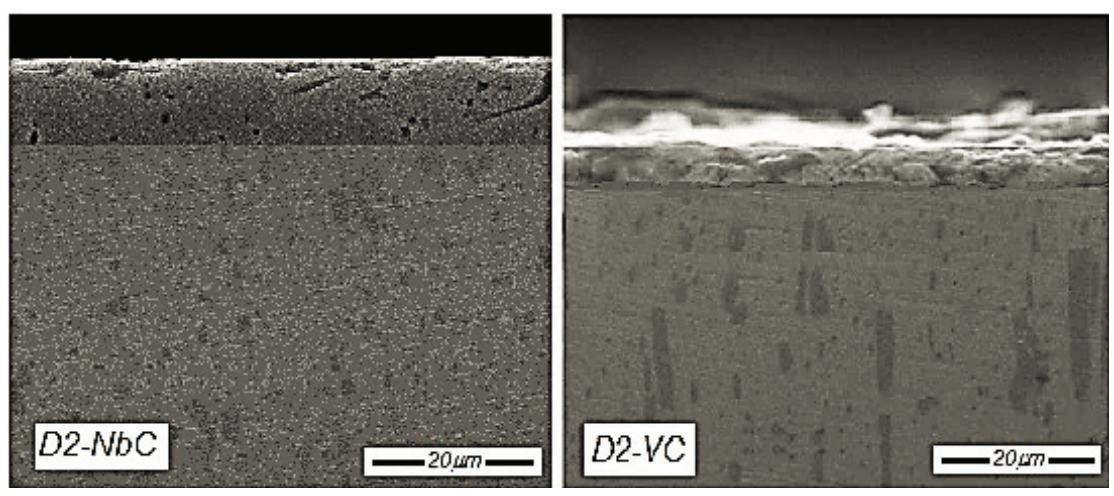

Figura 3. Micrografía SEM para los recubrimientos de carburo de niobio, a) $\mathrm{NbC}$ y b) carburo de vanadio VC sobre acero AISI D2

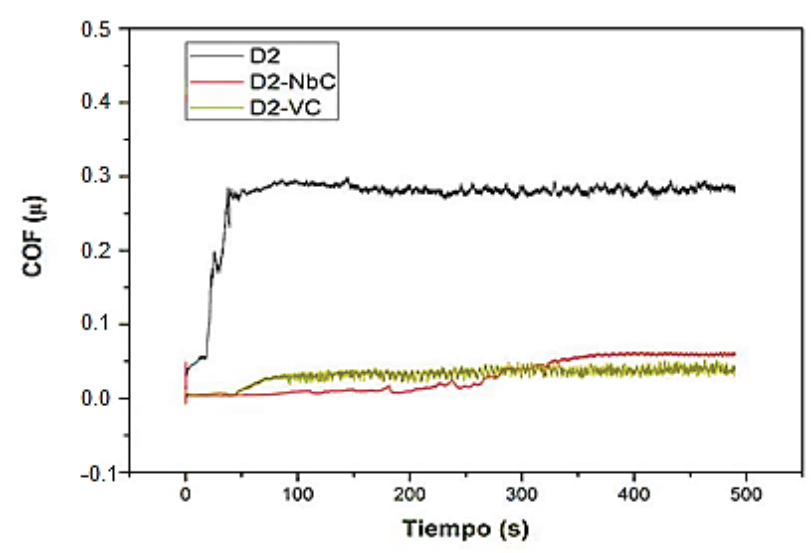

Figura 4. Comparación del coeficiente de fricción para recubrimientos de NbC y VC sobre acero AISI D2

El bajo coeficiente de fricción obtenido en los recubrimientos formados se puede asociar a la formación de átomos libres de $\mathrm{C}$ que se encuentran contaminando la superficie del recubrimiento en forma de grafito que se observa en la figura 5 con el excedente de $\mathrm{C}$ que favore- cen la formación de esta película y con ella la reducción del coeficiente de fricción; fenómenos similares se reportan en la literatura (Zhiwei et al., 2009). Se ha demostrado también la disminución del coeficiente de fricción debido a la formación de una película de baja resistencia al corte en la superficie de una película cerámica, lo cual también disminuye el coeficiente de fricción como se muestra en trabajos de recubrimientos TiN y DLC (Castillejo et al., 2011). Este comportamiento se ha reportado para recubrimientos de carburo de niobio (Chu Y Shen, 2008), donde los valores del coeficiente de fricción son cercanos a 0.1. Con las mismas técnicas de depósito se han reportado valores de coeficiente de fricción entre 0.1 y 0.4 (Sen y Sen, 2008; Sen, 2005). Otra causa que permite establecer la disminución en el coeficiente de fricción, es la naturaleza cerámica del recubrimiento debido a la disminución del tamaño del cristalito, lo que aumenta la densidad de dislocaciones y se refleja en el aumento de la dureza (Castillejo et al., 2014; Paunovic y Schlesinger, 1999; Oliver y Pharr, 1992; Niels, 2004) casi próxima a valores de dureza de
Figura 5. Análisis de EDS sobre recubrimientos de $\mathrm{NbC}$ y $\mathrm{VC}$ sobre acero AISI D2 indicando zona del substrato, zona de la interfase y la zona del recubrimiento que muestra un aumento de carbono hacia la superficie

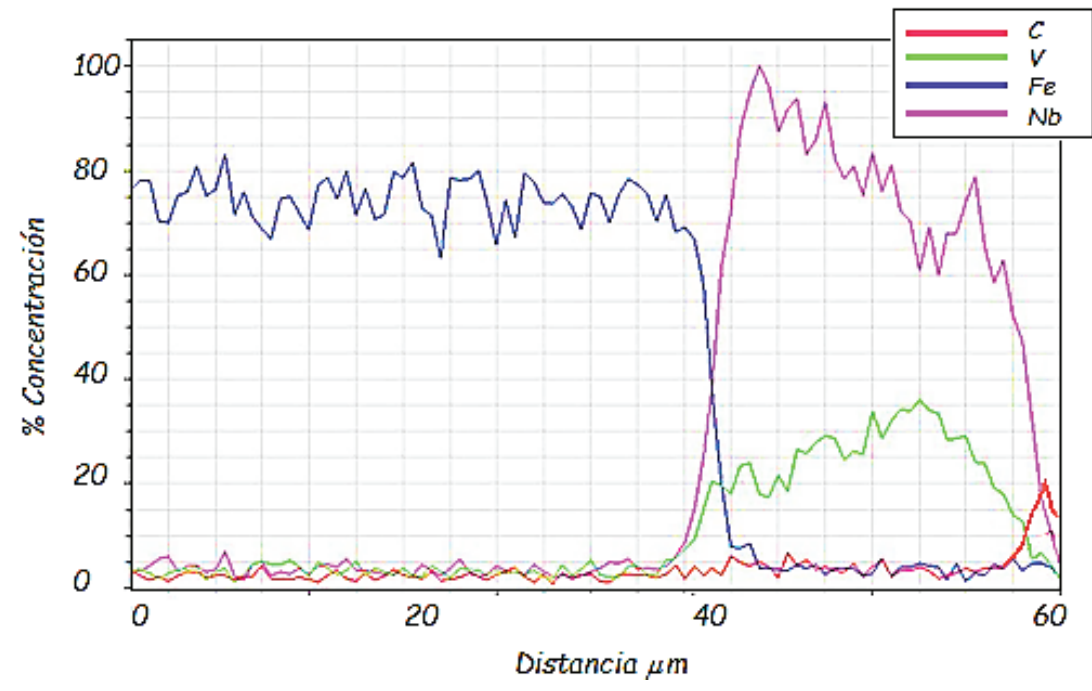



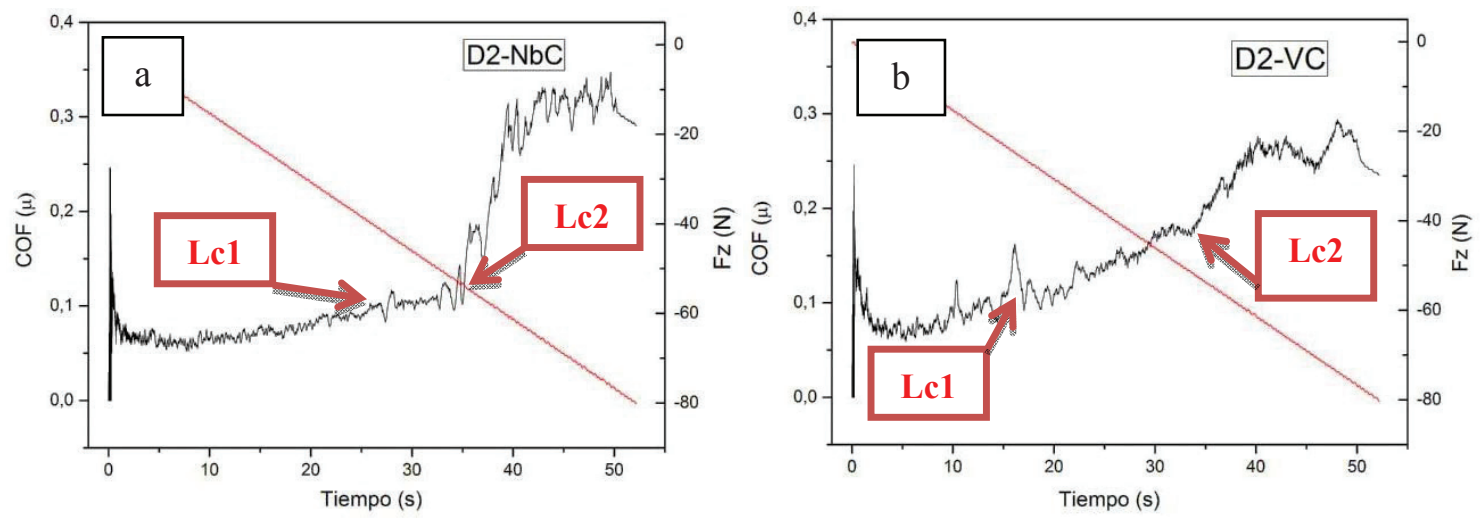

Figura 6. Prueba de rayado para recubrimientos de a) NbC y b) VC sobre acero AISI D2, relación de la fuerza aplicada (Fz, línea inclinada) y el coeficiente de fricción (COF)
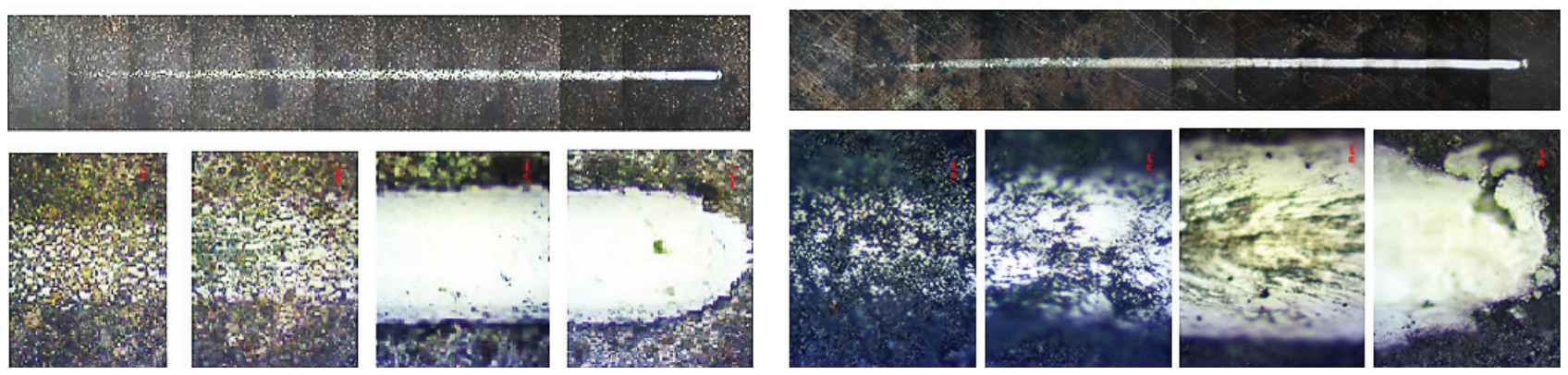

Figura 7. Huella de rayado para recubrimientos de: a) NbC y b) VC sobre acero AISI D2

recubrimientos de DLC y diamante, que han reportado otros investigadores (Trava et al., 2007).

La figura 6 muestra el resultado de un ensayo de adhesión para ambas muestras. Se identifica la carga inicial (Lc1), la fase de aplicación de la carga y el sitio donde la carga crítica (Lc2) genera falla y delaminación del recubrimiento. Para el recubrimiento D2-Nb, la falla inicial Lc1 comienza en torno de $41 \mathrm{~N}$ en un tiempo de 27 s evidenciando un tipo de falla cohesiva por el carácter duro del recubrimiento debido al desconchamiento (Bucking Spallation) en donde aún no hay desprendimiento, lo cual se observa en la figura 7a. La falla crítica Lc2 se produce en torno a $60 \mathrm{~N}$ en donde comienza la exposición del sustrato por la delaminación total del recubrimiento y una alteración en el comportamiento del COF con presencia de falla adhesiva (Sadumann et al., 2005; Sen y Sen, 2007).

De la misma manera para el recubrimiento D2-VC la falla inicial Lc1 se presenta en $20 \mathrm{~N}$ con pequeños desconchamientos que se evidencian en la figura $7 \mathrm{~b}$ y en donde se observan los mecanismos de desgaste, al final una falla de tipo adhesivo con exposición total del sustrato y remoción completa del recubrimiento cerca de $55 \mathrm{~N}$ en un tiempo de $36 \mathrm{~s}$ con propagación hacia los bordes (Gross Spallation) de la huella debido al carácter duro del recubrimiento prevista para este tipo de recubrimiento (Holmberg et al., 1998).

\section{Conclusiones}

Mediante la deposición de películas por la técnica DTR se fabricaron carburos de niobio y vanadio sobre aceros AISI D2 para usarse como recubrimientos con buen desempeño tribológico. Por medio del análisis de rayos $\mathrm{X}$ se observó que las capas producidas tienen una estructura cristalina FCC con las orientaciones mixtas, principalmente en las direcciones (111), (200) y (222). Para la caracterización tribológica usando el ensayo de esfera sobre disco se obtuvieron valores del coeficiente de fricción de 0.05 hasta 0.1 , este valor tiene una reducción considerable frente al coeficiente de fricción del acero D2 sin recubrir (superior a 0.3). Los resultados obtenidos en la prueba de rayado evidencian valores de carga crítica del orden de $55 \mathrm{~N}$ que corresponden a un sistema de alta adherencia con posibilidades de ser aplicado en elementos que estén sometidos a desgaste. Mediante microscopia de barrido se encontró que las capas crecieron uniformemente, con interfaces planas y 
definidas. Los valores de dureza fueron superiores a 23 GPa, en donde se evidencia un incremento en la dureza de 2 a 4 veces con relación al sustrato. La combinación de estos resultados permite recomendar la producción de recubrimiento carburos de $\mathrm{Nb}$ y de $\mathrm{V}$ con el sistema de deposición difusión termo reactiva para mejorar la durabilidad del acero AISI D2.

\section{Agradecimientos}

Este trabajo se realizó gracias al apoyo de la DIB-Universidad Nacional de Colombia, Colciencias a través del proyecto de Carburos Ternarios depositados con la técnica de DTR 1101-521-28337 y el Instituto Nacional de Investigaciones Espaciales del Brasil INPE, grupo Dimare.

\section{Referencias}

Arai T. Thermo reactive deposition diffusion process, en: Heat treating, 10a ed., volumen 4, Materials Park, OH: ASM International Handbook, 1991, pp.448

Arai T. Carbide coating process by use of molten borax bath in japan. J. Heat Treat., volumen 18 (número 2), 1979: 15-22.

Castillejo F., Marulanda D., Olaya J.J., Rodriguez O. Electrical furnace for producing carbide coatings using the thermoreactive deposition/diffusion technique. Dyna, volumen 78 (número 170), diciembre 2011: 192-197.

Castillejo F., Marulanda D., Olaya J. Estudio de recubrimientos de carburos ternarios de niobio-vanadio producidos sobre acero d2 usando la técnica de deposición por difusión termorreactiva. Revista LatinAm. Metal. Mat., volumen 34 (número 2), 2014: 230-239.

Chu K. y Shen Y.G. Mechanical and tribological properties of nanostructured TiN/TiBN multilayer films. Wear, volumen 265 (numeros 3-4), julio 2008: 516-524.

Holmberg K., Matthews A., Ronkainen H. Coatings tribology contact. Mechanisms and Surface Design, volumen 31 (número 98), 1998a: 107-120.

Niels H. Hall-Petch relation and boundary strengthening. Scr. Mater, volumen 51 (número 8), 2004: 801-806.

Oliveira C.K.N., Muñoz-Riofano R.M., Casteletti L.C. Evaluation of hard coatings obtained on AISI D2 steel by thermoreactive deposition treatment. Surface \& Coatings Technology, volumen 201, 2006a: 1880-1885.
Oliveira C.K.N., Muñoz-Riofano R.M., Casteletti L.C., Micro-abrasive wear test of niobium carbide layers produced on AISI H13 y M2 steels. Surface and Coatings Technology, volumen 200, 2006b: 5140-5144.

Oliveira C.K.N., Muñoz-Riofano R.M., Casteletti L.C. Formation of carbide layers on AISI H13 y D2 steels by treatment in molten borax containing dissolved both $\mathrm{Fe}-\mathrm{Nb}$ and Fe-Ti powders, Materials Letters, volumen 59, 2005: 1719-1722.

Oliver W.C. y Pharr G.M. An inproved technique for determining hardness and elastic modulus using load and displacement sensing identation experiments. J. Mater Res., volumen 7 (número 6), 1992: 1564-1583.

Patiphan J. y Prasonk S. Influence of reducer on coating of steel with vanadium carbide by TD process, Department of Metallurgical Engineering, Chulalongkom University, Bangkok, Thailand.

Paunovic M., Schlesinger M. Procesamiento de materiales integrales, Nueva York, John Wiley \& Sons, USA, 1999.

Peter A., De Paula J. Physical Chemistry, 9ª ed., parte 1, capítulo 4, Nueva York, W.H. Freeman and Company, 2012.

Saduman S. A study on kinetics of CrxC-coated high-chromium steel by thermo-reactive diffusion technique. Vacuum, volumen 79, 2005: 63-70.

Saduman S., Sen U., Cuma B. The Growth Kinetics of Borides Formed on Boronized AISI 4140. Vacuum, volumen 77, 2005: 195202.

Sen U. y Sen S. Characterization of niobium carbonitride on AISI D2 steel. Materials Science Forum, volumen 554, 2007: 213-217.

Sen U. Kinetics of niobium carbide coating produced on AISI 1040 steel by thermo-reactive deposition technique. Materials Chemistry and Physics, volumen 86 (número 1), 2004: 189-194.

Sen U. Friction and wear properties of thermo-reactive diffusion coatings against titanium nitride coated steels. Materials and Design, volumen 26, 2005: 167-174.

Sen S. y Sen U. Sliding wear behavior of niobium carbide coated AISI 1040 steel. Wear, volumen 264 (números 3-4), febrero 2008: 219-225.

Sen U. Wear properties of niobium carbide coatings performed by pack method on AISI 1040 steel. Thin Solid Films, volumen 483 (números 1-2), julio 2005: 152-157.

Trava-Airoldi V.J., Bonetti L.F., Capote G., Fernandes J.A., Blando E., Hübler R., Radi P.A., Santos L.V., Corat E.J. DLC film properties obtained by a low cost and modified pulsed-DC discharge. Thin Solid Films, volumen 516, 2007: 272-276.

Zhiwei Z., Ying L., Hong C., Hongjuan Z., Jinwen Y., Shengji G., Mingjing T. Journal of Alloys and Compounds, volumen 468, 2009: 58-63. 


\begin{abstract}
Este artículo se cita:
Citación estilo Chicago

Ramírez-Ramos, Marco Antonio, John Jairo Olaya-Florez, Vladimir Jesús Airoldi-Trava. Evaluación tribológica de recubrimientos de NbC y VC sobre acero AISI D2 producidos por la técnica deposición difusión termo-reactiva. Ingeniería Investigación y Tecnología, XVI, 02 (2015): 287-294.

\section{Citación estilo ISO 690}

Ramírez-Ramos M.A., Olaya-Florez J.J., Airoldi-Trava V.J. Evaluación tribológica de recubrimientos de NbC y VC sobre acero AISI D2 producidos por la técnica deposición difusión termo-reactiva (DTR). Ingeniería Investigación y Tecnología, volumen XVI (número 2), abril-junio 2015: 287-294.
\end{abstract}

\section{Semblanza de los autores}

Marco Antonio Ramírez-Ramos. Profesional en ingeniería con maestría en materiales y procesos de la Facultad de Ingeniería Mecánica y Mecatrónica de la Universidad Nacional de Colombia UNAL. Actualmente estudia el doctorado en la Universidad Federal de São Paulo UNIFESP con investigación en desarrollo en el Instituto Nacional de Investigaciones Espaciales del Brasil INPE. Sus temas de investigación son: modificación de superfícies con recubrimientos duros sobre aceros en general y estudio tribológico de los recubrimientos.

Jhon Jairo Olaya-Florez. Profesional en ingeniería metalúrgica con maestría en materiales y procesos por la Facultad de Ingeniería Mecánica y Mecatrónica de la Universidad Nacional de Colombia. Obtuvo el doctorado en materiales por la Universidad Autónoma de México UNAM y actualmente es profesor asociado de la UNAL.

Vladimir Jesus Trava-Airoldi. Profesional en física por el Instituto de Física de la Universidad de São paulo USP, tiene la maestría y el doctorado en física por el instituto Tecnológico aeronáutico ITA y el posdoctorado en el Jet Propulsion Laboratory NASA/USA y California Institute of Technology CALTECH/USA. Actualmente es investigador senior en el Instituto Nacional de Investigaciones espaciales del Brasil y profesor de posgrados. 